\title{
Study of Relation Between Disability and Symptomatology with Recovery in Schizophrenia
}

\author{
Bindoo S. Jadhav ${ }^{1} \cdot$ Sunitha Shanker $^{1} \cdot$ Riddhish K. Maru $^{1} \cdot$ Bharat R. Shah $^{1}$ \\ Hemangee S. Dhavale ${ }^{1}$
}

Received: 3 March 2015/Accepted: 24 August 2015/Published online: 9 September 2015

(C) Springer India Pvt. Ltd. 2015

\begin{abstract}
There is a great paucity of studies on recovery in schizophrenia in India. Hence, this study was undertaken to assess (1) the relationship between disability and stages of recovery and (2) the relationship between disability, recovery and symptomatology. Data was collected from a convenience sample of 50 schizophrenia patients using semi structured proforma, Positive and Negative Syndrome Scale (PANSS), Indian Disability Evaluation and Assessment Scale and Stages of Recovery Instrument (STORI), and subjected to appropriate statistical analysis. In the sample studied, stage of moratorium of STORI had significant positive correlation, while stage of preparation and growth had significant negative correlation with disability. Stage of moratorium had significant positive correlation with all subscales of PANSS while stages of awareness, preparation and rebuilding and growth had significant negative correlation with all subscales of PANSS. This suggests that there is a significant relationship between level of functioning, psychopathological symptoms and person's ability to pursue recovery.
\end{abstract}

Keywords Schizophrenia $\cdot$ Stages of recovery · Disability $\cdot$ Symptomatology

Bindoo S. Jadhav

blmaru@gmail.com

1 Department of Psychiatry, K.J.Somaiya Medical College and Research Centre, Somaiya Ayurvihar, 292/A-10, 'Amarjyoti Apartments', Gulmohar Lane, Sion, Chunabhatti, Mumbai, Maharashtra 400022, India

\section{Introduction}

Recovery is the process by which a person overcomes the challenges presented by mental illness to live a life of meaning and purpose. Hence, recovery orientation to psychiatric illness involves a "redefinition of one's illness as only one aspect of a multidimensional sense of self, capable of identifying, choosing and pursuing personally meaningful goals and aspirations" [1]. Liberman [2] provides the most specific guidelines, they prescribe a Brief Psychiatric Rating Scale (BPRS) score of 4 or less (clinically non-significant) on all positive and negative symptoms items, at least half time work or school, person capable of independent management of funds and medication, and once a week socializing with peers for a period of 2 years.

According to William Anthony [3], recovery is "a deeply personal, unique process of changing one's attitudes, values, feelings, goals, skills and/or roles. It is a way of living a satisfying, hopeful and contributing life even with limitations caused by the illness. Recovery involves the development of new meaning and purpose in one's life as one grows beyond the catastrophic effects of mental illness".

Recovery from mental illness is not the same as cure. The recovery literature challenges the "traditional" or medical understanding that recovery means cure or absence of symptoms because it deals with only a part of the impact of severe mental illness (i.e. the symptoms). Recovery oriented services have expanded the concept of service outcome to include such dimensions as self-esteem, adjustment to disability, empowerment, and self-determination. From a recovery perspective, these successful outcomes may lead to the growth of new meaning and purpose in the person's life. This gives us an understanding of how much effort goes in designing a recovery program for people with this disorder. 
Disability is a major contributor to the global burden of disease. Psychiatric disorders, especially schizophrenia, are one of the leading causes of disability worldwide. According to the Global Burden of Disease Study, schizophrenia causes a high degree of disability, which accounts for $1.1 \%$ of the total disability adjusted life years (DALYs) and $2.8 \%$ of years lived with disability (YLDs). In the World Health Report, schizophrenia is listed as the 8th leading cause of DALYs worldwide in the age group 15-44 years [4]. Study of disability associated with mental disorders, therefore, becomes a matter of prime importance.

Residual disability and poor quality of life continue even after completion of symptom-linked treatment. To assess and quantify the disability will help to understand, plan and expect accordingly and appropriately for management and rehabilitation of these patients.

There is no available Indian literature on disability and recovery to the best of our knowledge. Efforts in promoting recovery and decreasing disability, in these patients has to start with identifying the extent to which our patient population is suitable for it. This study was undertaken keeping these goals in mind.

\section{Aims and Objectives}

The primary objective of the current study was to assess the relationship between disability and the stages of recovery of schizophrenia.

The secondary objectives were to study the relationship between disability, stages of recovery of schizophrenia and symptomatology.

\section{Materials}

- Semi-structured proforma a specially designed intake form to record patient related socio demographic variables and illness related information of each subject.

- Positive and Negative Syndrome Scale (PANSS) [5] PANSS is a comprehensive and standardized scale used for measuring symptom severity of patients with schizophrenia and other psychotic disorders. It was published in 1987 by Stanley Kay, Lewis Opler, and Abraham Fiszbein. The PANSS measurement derives from behavioral information plus a four phase 35-45 min clinical interview, followed by seven point ratings on 30 symptoms, for which each item and each level of symptom severity are defined. It has three subscales i.e. general psychopathology, positive and negative subscales.

- Stages of Recovery Instrument (STORI) [6] The STORI consists of 50 items, presented in ten groups of five.
Each item is rated by the patient on a Likert scale of $0-5$, where 0 represents not at all true and 5 represents completely true. The questionnaire asks how patient feels about one's life and oneself, since the illness. Individual items within each group represent the stage of recovery which is stage of Moratorium, Awareness, Preparation, Rebuilding and Growth. There is no "Total" score. The stage with the highest total is taken as the person's stage of recovery.

- Indian Disability Evaluation and Assessment Scale (IDEAS) [7] The Rehabilitation Committee of Indian Psychiatric Society developed this scale. It has four items: Self Care, Interpersonal Activities (Social Relationships), Communication and Understanding, and Work. Each item is scored between 0 and 4, i.e. from no to profound disability; adding scores on four items gives the 'total disability score'. Global disability score is calculated by adding the 'total disability score' and MI2Y score (months in 2 years - a score ranging between 1 and 4, depending on the number of months in the last 2 years the patient exhibited symptoms). Global disability score of:

$$
\begin{aligned}
& 0-\text { No disability }=0 \% \\
& 1-7-\text { Mild disability }=<40 \% \\
& 8-13-\text { Moderate disability } \\
& 14-19-\text { Severe disability } \\
& 20-\text { Profound disability }
\end{aligned}
$$

The alpha value of the scale is 0.8682 , indicating good internal consistency between the items. It also has good criterion validity.

\section{Inclusion Criteria}

1. Patients of chronic schizophrenia with duration of illness $>2$ years.

2. Patients in age group of 18-60 years.

\section{Exclusion Criteria}

1. Patients with any other co-morbid psychiatric illness.

2. Patients of chronic schizophrenia having relapse in last 6 months.

\section{Methods}

This is a cross-sectional co relational study. The sample of the study is $\mathrm{N}=50$. The pool of participants diagnosed with schizophrenia as per DSM-IV-TR attending the psychiatric outpatient department of a tertiary teaching hospital 
in Mumbai and fulfilling the inclusion and exclusion criteria were recruited in the study through convenience sampling.

After seeking approval from the Ethics Committee, and taking written informed consent, the study was started. Semi-structured proforma was administered to patients to collect socio-demographic data and illness related information. The tools (PANSS, IDEAS, STORI) were then applied on all the patients at one point in time to assess symptomatology, stage of recovery and disability respectively. All the tools applied were administered by psychiatrists with significant clinical and academic experience.

SPSS v. 20 was used to statistically analyze the data. Since the sample was not normally distributed, non-parametric statistical tool of Spearman's Rank correlation was used to seek the relationship between the variables studied. The demographic data was studied by applying the descriptive statistical tools.

\section{Results}

In our study, 50 patients with a diagnosis of schizophrenia were included.

Table 1 shows the socio-demographic profile of the sample studied.

In our study, $33(66 \%)$ patients were in the age group of $18-40$. With regards to gender distribution, $42 \%$ of the sample consisted of males and $58 \%$ were females. In the sample studied, 22 (44\%) patients had secondary education and $28(56 \%)$ were married. Also, $16 \%$ of the sample was self-employed, $26 \%$ were unemployed and $42 \%$ were housewives.

In the correlation computed, no significant results were obtained between the demographical variables and the stages of recovery and disability.

Graph 1 shows the distribution of presence of family history of psychiatric illness in the sample studied. $68 \%$ of the patients studied had a positive family history of schizophrenia or other psychiatric illness.

As shown in Graph 2, $82 \%$ of the sample studied had a diagnosis of paranoid schizophrenia.

The highest range of scores in the PANSS has been in the general psychopathology i.e. GPS (mean $=22.32$ ) subscale followed by negative (mean $=12.51)$ and positive (mean $=10.23$ ) symptoms subscale, as is seen in Table 2. This pattern indicates that problems related to general psychopathology was maximum in the sample studied.

One can observe a relatively diffuse distribution of scores of stages of recovery in Table 3 . The stage of moratorium is observed to have the smallest mean score which clearly indicates that majority of the sample studied is in a higher stage of recovery.
Table 1 Socio-demographic profile of the sample

\begin{tabular}{|c|c|c|}
\hline Socio-demographic variables & Total patients $\mathrm{N}=50$ & $\%$ \\
\hline \multicolumn{3}{|l|}{ Age } \\
\hline $18-40$ years & $33(66 \%)$ & 66 \\
\hline $41-60$ years & $16(32 \%)$ & 32 \\
\hline$>60$ years & $01(2 \%)$ & 02 \\
\hline \multicolumn{3}{|l|}{ Sex } \\
\hline Male & $21(42 \%)$ & 42 \\
\hline Female & $29(58 \%)$ & 58 \\
\hline \multicolumn{3}{|l|}{ Education } \\
\hline Illiterate & $04(8 \%)$ & 08 \\
\hline Primary & $09(18 \%)$ & 18 \\
\hline Secondary & $22(44 \%)$ & 44 \\
\hline Higher secondary & $04(8 \%)$ & 08 \\
\hline Graduate & $11(22 \%)$ & 22 \\
\hline Post-graduate & - & - \\
\hline \multicolumn{3}{|l|}{ Marital status } \\
\hline Single & $16(32 \%)$ & 32 \\
\hline Married & $28(56 \%)$ & 56 \\
\hline Separated & $03(6 \%)$ & 06 \\
\hline Divorced & $02(4 \%)$ & 04 \\
\hline Widow & $01(2 \%)$ & 02 \\
\hline \multicolumn{3}{|l|}{ Occupation } \\
\hline Self employed & $08(16 \%)$ & 16 \\
\hline Service & $07(14 \%)$ & 14 \\
\hline House-wife & $21(42 \%)$ & 42 \\
\hline Unemployed & $13(26 \%)$ & 26 \\
\hline Student & $01(2 \%)$ & 02 \\
\hline
\end{tabular}

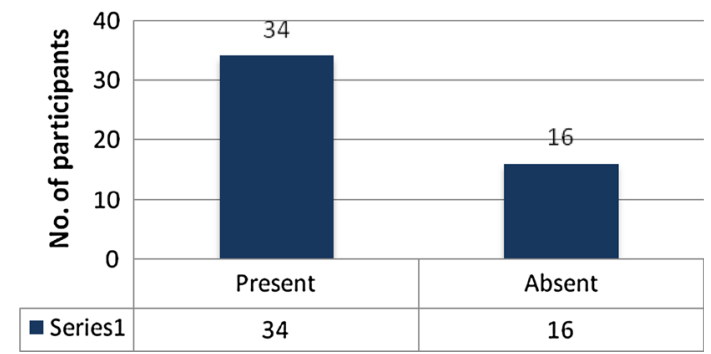

Graph 1 Presence of family history of psychiatric illness

Table 4 shows the correlation between the stages of recovery and IDEAS while Table 5 shows the relationship between symptomatology and stages of recovery.

The first stage where the patient withdraws and feels a profound sense of loss and hopelessness is called Moratorium. It is observed that there is a positive correlation between the stage of moratorium and disability $(r=0.33$, $\mathrm{p}<0.05)$. This indicates that as disability increases patient 
is more withdrawn and experiences a sense of hopelessness. Also, this stage has a significant positive correlation with all the sub-scales of PANSS, indicating that as the symptoms increase, the patient experiences the stage of moratorium.

The second stage of recovery is called Awareness where the person begins to have hope about the future and gradually believes in the possibility that one can lead a

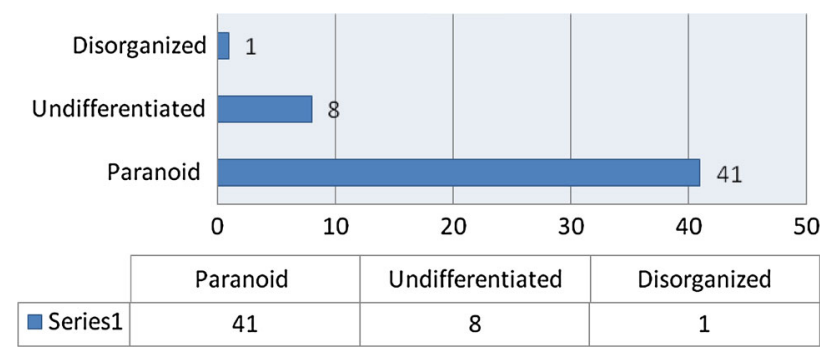

Graph 2 Type of Schizophrenia

Table 2 Shows the mean scores on PANSS

\begin{tabular}{ll}
\hline PANSS & Mean scores \\
\hline Positive & 10.23 \\
Negative & 12.51 \\
General psychopathology & 22.32 \\
\hline
\end{tabular}

Table 3 Shows the distribution of mean scores on the scale of STORI

Table 4 Correlations of stages of recovery with IDEAS

Table 5 Correlations of stages of recovery with PANSS

$* \mathrm{p}<0.05$ fulfilling life. The stage of awareness does not have a significant relationship with disability $(\mathrm{r}=-0.183, \mathrm{~ns})$. There is a negative correlation; however the findings are not significant. This stage has a significant negative correlation with the GPS $(\mathrm{r}=-0.609, \mathrm{p}<0.01)$ and negative symptoms $(\mathrm{r}=-0.495, \mathrm{p}<0.01)$ subscale of PANSS.

There is a significant negative correlation observed between the stage of preparedness and disability $(\mathrm{r}=-0.310, \mathrm{p}<0.05)$. The point where a person with schizophrenia begins to work on developing skills towards his recovery based on his strengths and weaknesses is the stage of Preparedness. These results indicate that as the affected person's level of functioning increases, his level of disability decreases (and vice versa). Results also show that there is a significant negative correlation with the positive symptoms $(\mathrm{r}=-0.335, \mathrm{p}<0.05)$, negative symptoms $(\mathrm{r}=-0.361, \mathrm{p}<0.05)$ and GPS $(\mathrm{r}=-0.684, \mathrm{p}<0.01)$ subscale, suggesting that an increase in symptoms of the illness is related to a decrease in the ability to prepare for recovery.

Significant correlation was not observed between the stage of 'rebuilding' and IDEAS ( $\mathrm{I}=-0.201, \mathrm{p}=\mathrm{ns}$ ). When the person with schizophrenia begins to actively work towards a positive identity and setting meaningful goals, he/she is said to be in the stage of Rebuilding. The negative correlation, even though not significant, indicates that as the person progresses towards the fourth stage of recovery, there is a decrease in his disability. It is observed that 'rebuilding' stage has significant negative correlations with the positive symptoms $(\mathrm{r}=-0.402$, $\mathrm{p}<0.05)$, negative symptoms $(\mathrm{r}=-0.363, \mathrm{p}<0.05)$ and GPS ( $r=-0.651, p<0.01)$ subscales. This suggests that when symptoms increase, the patient is less likely to be able to set goals.

There is a significant negative correlation between the stage of 'growth' and disability ( $\mathrm{r}=-0.336, \mathrm{p}<0.05)$. Growth is the last stage of recovery in which a person with

\begin{tabular}{llllll}
\hline & Moratorium & Awareness & Preparation & Rebuilding & Growth \\
\hline IDEAS & $0.328^{*}$ & -0.183 & $-0.310^{*}$ & -0.201 & $-0.336^{*}$
\end{tabular}

\begin{tabular}{llllll}
\hline & Moratorium & Awareness & Preparation & Rebuilding & Growth \\
\hline PANSS (Neg) & $0.401^{*}$ & -0.280 & $-0.361^{*}$ & $-0.363^{*}$ & $-0.411^{*}$ \\
PANSS (Pos) & $0.525^{* *}$ & $-0.495^{* *}$ & $-0.335^{*}$ & $-0.402^{*}$ & $-0.411^{* *}$ \\
GPS & $0.855^{* *}$ & $-0.609^{* *}$ & $-0.684^{* *}$ & $-0.651^{* *}$ & $-0.721^{* *}$ \\
\hline
\end{tabular}

$* \mathrm{p}<0.05$

$* * \mathrm{p}<0.01$ 
Table 6 Correlation of IDEAS with PANSS

\begin{tabular}{llll}
\hline & Positive symptoms & Negative symptoms & General psychopathology \\
\hline IDEAS & 0.158 & 0.194 & $0.525 * *$ \\
\hline$* * \mathrm{p}<0.01$ & & &
\end{tabular}

schizophrenia begins to live a meaningful life, proactively managing his illness with resilience. Results show that there is a significant negative correlation with the positive symptoms $(\mathrm{r}=-0.411, \mathrm{p}<0.01)$, negative symptoms $(\mathrm{r}=-0.411, \mathrm{p}<0.05)$ and GPS $(\mathrm{r}=-0.721, \mathrm{p}<0.01)$ subscales. This indicates that as the symptoms of the illness decrease, the possibility of meaningful recovery increases.

Table 6 shows that there is a significant positive relationship between the general psychopathology scale of PANSS and disability as measured by IDEAS $(r=0.525$, $\mathrm{p}<0.01)$. However, the relationship between the positive $(\mathrm{r}=0.158, \mathrm{p}=\mathrm{ns})$ and negative $(\mathrm{r}=0.194, \mathrm{p}<\mathrm{ns})$ symptoms subscales, and disability is not significant.

\section{Discussion}

This study was conducted as an endeavor to identify which stage of recovery most of our schizophrenia patients are in and some of the significant factors related to it. The following sections of the paper will highlight the main findings and discuss them in the light of previous global findings and experience in India.

When we observe the distribution of the sample in the stage of recovery, it clearly indicates that lesser number of participants were in the moratorium stage. This could probably be due to the fact that most of them were relatively well functioning and did not have any comorbid psychiatric conditions. In the stage of "preparation' and 'rebuilding' the minimum score obtained by the subjects was relatively higher in comparison to the other stages, suggesting that the preparatory stage of recovery is where the subjects in the sample are probably leaning towards.

This study looked at the relationship between disability and specific stage of recovery. The pattern of the results obtained indicate that patients in the first stages of recovery, where they are feeling helpless, have higher levels of disability and as they progress towards the stage of growth, there is a decrease in the disability. Even though the correlation is relatively weak, this pattern clearly indicates the significant role of disability in recovery from schizophrenia. A study by Alptekin et al. [8] has shown that disability has been affected by characteristics like age of onset, duration of illness, severity and type of symptoms, duration of untreated illness, cognitive deterioration and intellectual functioning. Many of these factors can also significantly affect the recovery process in a person with schizophrenia. This shows that disability and the stage of recovery in a person in schizophrenia will be most often inter-linked.

Disability in people with schizophrenia is pervasive, affecting social, vocational, and residential domains. Impairments in these domains are present over the course of illness and are evidenced by reductions in achieving functional milestones common in the general population, such as independent living, marriage, and employment. As treatment goals shift from symptom remission to recovery in these functional areas, clinicians must begin to understand the dimensions of functional impairment, increase familiarity with assessments of functional disability, and understand the predictors of functioning in order to help recovery in schizophrenia patients [9].

The study results show that there is a significant relationship between psychopathological symptoms and the person's ability to have a meaningful recovery. It is also seen that general psychopathology scale has maximum impact on all the stages of recovery. There is also a significant relationship observed between the general psychopathology scale and disability.

Research in schizophrenia has clearly proved that one of the most disabling factors in this disorder is the psychopathology associated with the disorder and they affect the person's level of functioning $[10,11]$. This means that increased symptoms will result in disability and prevent the person from achieving meaningful recovery. The above finding brings to light the importance of a rigorous pharmacological treatment protocol along with well designed psychological interventions that will address the symptoms in the illness with swift efficiency.

\section{Conclusions}

Of the 50 patients with schizophrenia studied, majority of the patients were in the age group of 18-40 years. and married with a slight female preponderance. There were no significant findings in the correlation computed between the demographical variables and the stages of recovery and disability. Stage of moratorium had significant positive correlation with disability while stages of preparation and growth had significant negative correlation with disability. The results indicate the need for a good assessment plan which will aim to seek the level of disability experienced by the patient in each area of his life, the disorder related 
symptoms that are causing the specific disabilities and how it is affecting his/her daily living. And accordingly plan intervention programs and techniques that can be translated and generalized in daily life.

Stage of moratorium had significant positive correlation while stages of awareness, preparation, rebuilding and growth had significant negative correlation with psychopathology. These suggest that psycho-pharmacological interventions followed by well planned individually tailored psycho-social interventions to decrease the psychopathological symptoms will assist the recovery process.

\section{Limitations}

- The sample size was relatively smaller and the study was cross sectional in design.

- It was a hospital based study, hence findings of the study cannot be generalized to community.

- Paucity of research in this area did not allow the authors to have a detailed theoretical backup for the generalizations made.

- The study has not studied in detail the sub-domains of the variables which would have yielded richer data. This was not undertaken because this was not the objective of this preliminary study. Subsequent studies in this area will endeavor to understand the finer details in the area of recovery and schizophrenia.

\section{Recommendations}

Schizophrenia, being a heterogeneous disorder, demands heterogeneous interventions. Whereas it is important to treat the symptoms of the illness with medication, equally vital are skills training intervention in promoting independence and decreasing disability. It can be hypothesized that when a person is able to acquire skills, the lack of which are disabling him/her, it will promote the feelings of empowerment and high self efficacy which itself will go a long way in helping the person march towards the stage of growth.

These scores have highlighted the need for a longitudinal community based study with a more heterogeneous sample to identify the level of disability and stage of recovery our population with schizophrenia is in. This will help us to plan further effective interventions.

\section{Compliance with Ethical Standards}

Conflict of Interest All the five authors of this manuscript declare that they have no conflict of interest.

\section{References}

1. Davidson L. Recovery, self management and the expert patientchanging the culture of mental health from a UK perspective. J Ment Health. 2005;14:25-35.

2. Liberman RP, Kopelowicz A, Ventura J, Gutkind D. Operational criteria and factors related to recovery from schizophrenia. Int Rev Psychiatry. 2002;14:256-72.

3. Anthony William. Recovery from mental illness: the guiding vision of the mental health service system in the 1900's. Psychosoc Rehabil J. 1993;16(4):11-23.

4. Rossler W, Salize HJ, van Os J, Riecher-Rossler A. Size of burden of schizophrenia and psychotic disorders. Eur Neuropsychopharmacol. 2005;15(4):399-409.

5. Kay SR, Fiszbein A, Opler LA. The positive \& negative syndrome (PANSS) scale. Schizophr Bull. 1987;13:261-76.

6. Andresen R, Caputi P, Oades L. The Stages of Recovery Instrument: development of a measure of recovery from serious mental illness. Aust NZ J Psychiatry. 2006;40:972-80.

7. Indian disability assessment and evaluation scale. Rehabilitation Committee of Indian Psychiatric Society; 2000.

8. Alptekin K, Erkoc S, Gogus AK, Kultur S, Mete L, Ucok A, et al. Disability in schizophrenia: clinical correlates and prediction over 1-year follow-up. J Psychiatry Res. 2005;135:103-11.

9. Harvey PD. Disability in schizophrenia: contributing factors and validated assessments. J Clin Psychiatry. 2014;75(suppl 1):15-20.

10. Heinrich W, Ammari N, Miles A, McDermidVaz S, Chopov B. Psychopathology and cognition in divergent functional outcomes in schizophrenia. Schizophr Res. 2009;106:46-51.

11. Gaite L, Vázquez-Barquero JL, Herrán A, Thornicroft G, Becker $\mathrm{T}$, Sierra-Biddle $\mathrm{D}$, et al. Main determinants of global assessment of functioning score in schizophrenia: a European multicenter study. Compr Psychiatry. 2005;46:440-6. 